\title{
Meta
}

Journal des traducteurs

Translators' Journal

\section{KRÁlOVÁ, Jana y CUENCA DROUHARD, Miguel José (2013): Jiří \\ Levý: una concepción (re)descubierta. Soria: Diputación Provincial de Soria, 179 p.}

\section{Petra Vavroušová}

Volume 60, numéro 3, décembre 2015

URI : https://id.erudit.org/iderudit/1036149ar

DOI : https://doi.org/10.7202/1036149ar

Aller au sommaire du numéro

Éditeur(s)

Les Presses de l’Université de Montréal

ISSN

0026-0452 (imprimé)

1492-1421 (numérique)

Découvrir la revue

Citer ce compte rendu

Vavroušová, P. (2015). Compte rendu de [KRÁLOVÁ, Jana y CUENCA

DROUHARD, Miguel José (2013): Jiř́ Levý: una concepción (re)descubierta. Soria:

Diputación Provincial de Soria, 179 p.] Meta, 60(3), 644-645.

https://doi.org/10.7202/1036149ar d'utilisation que vous pouvez consulter en ligne.

https://apropos.erudit.org/fr/usagers/politique-dutilisation/ 
almost two page-long paragraph from page 18 to 20 stands out). At times, what are presented as back translations are somewhat confusing for the reader due to their ungrammaticality, seeming to randomly reflect some structural features of the source language but not others ("With the in this special report investigated capture and storage [...]," p. 29; "[...] and him enables to procure some meat fresh [...]," p. 82). As no commonly accepted standards for interlinear/gloss translations exist, these examples could have been made more accessible by a conventional gloss as standard in linguistics or a grammatical translation, especially as word order does not seem particularly relevant to the arguments made here.

A negative point about the paperback version of this book, especially given the reputation of its publisher, is that the inside margins are so small that the book cannot be read comfortably without forcing it apart at the spine or pressing it flat. This is a drawback especially for an academic book which often needs to be placed on a table and referred to while writing or typing rather than being held continuously.

In sum, however, the great achievement of the volume is that it provides an overview of translation studies and the various disciplines with which it interacts, as well as the angles from which it can be approached, making this book a treasure trove for researchers and students embarking on projects in the field.

MARIO BISIADA Universitat Pompeu Fabra, Barcelona, Spain

\section{REFERENCES}

BAKer, Mona (2009): Translation studies. In: Mona BAKER and Gabriela SAldANHA, eds. Routledge Encyclopedia of Translation Studies. London: Routledge, 277-280.

Bassnett, Susan and Lefevere, André (1990): Translation, History and Culture. London: Pinter Publishers.

Becher, Viktor (2011): Von der Hypotaxe zur Parataxe: Ein Wandel im Ausdruck von Konzessivität in neueren populärwissenschaftlichen Texten. In: Eva Breindl, Gisella Ferraresi and Anna Volodina, eds. Satzverknüpfungen. Zur Interaktion von Form, Bedeutung und Diskursfunktion. Berlin: de Gruyter, 277-280.

BISIADA, Mario (2013a): Changing conventions in German causal clause complexes: A diachronic corpus study of translated and non-translated business articles. Languages in contrast 13(1):127.

BisiadA, Mario (2013b): From Hypotaxis to Parataxis: An Investigation of English-German Syntactic Convergence in Translation. Doctoral thesis, unpublished. Manchester: University of Manchester.
BoAse-BeIER, Jean (2011): A Critical Introduction to Translation Studies. London: Continuum.

Schjoldager, Anne, Gottlieb, Henrik and KlitGARD, Ida (2008): Understanding Translation. Aarhus: Academica.

Snell-Hornby, Mary (1988): Translation Studies: An Integrated Approach. Amsterdam: John Benjamins.

Králová, Jana y Cuenca Drouhard, Miguel José (2013): Jiř́i Levý: una concepción (re)descubierta. Soria: Diputación Provincial de Soria, $179 \mathrm{p}$.

Cincuenta años después de la publicación de la obra clave del filólogo checo Jiří Levý (1926-1967), Umění překladu [El arte de la traducción] (1963), aparece una antología española que brinda al mundo académico hispanohablante la oportunidad de conocer las ideas y concepciones fundamentales de este teórico checo de la traducción. Las aportaciones procedentes del ámbito eslavo, el checo incluido, quedan ignoradas o poco descubiertas en la traductología internacional; en efecto, Levý optó por utilizar su lengua materna para formular sus tesis principales, ahí radica seguramente el desconocimiento de sus trabajos. Los profesores Jana Králová y Miguel José Cuenca Drouhard, de la Univerzita Karlova v Praze [Universidad Carolina de Praga], han sabido recopilar cuidadosamente los textos más representativos y esenciales de Levý y verterlos al español. Asimismo, cabe destacar el prólogo de Miguel Ángel Vega Cernuda que caracteriza el pensamiento de Levý como uno de los pensamientos más originales de la traducción.

El libro consta de una introducción y cinco capítulos. La introducción pone de manifiesto las raíces metodológicas del autor que parten del Círculo Lingüístico de Praga y respeta el orden de la primera aparición de sus trabajos. Entre las contribuciones de Levý destacan, ante todo, los dos siguientes postulados: la atención a la perspectiva histórica de la traducción y la consideración del ejercicio de la traducción como elemento integrante del sistema literario de una lengua. Asimismo, Levý se fija en la figura del traductor que no deja de evolucionar y constituye el elemento activo del proceso de la traducción.

El primer capítulo ofrece al lector español el prólogo a la primera edición y el epílogo de České teorie prekladu [Teorías checas de la traducción] (1956) que ponen énfasis en la especificidad de la evolución de la traducción checa, vinculada estrechamente a las necesidades inmediatas de la vida nacional, exigencias de carácter, ante todo, estético e ideológico. La cuestión estética gira en torno al novedoso "sistema de opiniones estéticas" 
de cada época, inclinándose bien hacia la fidelidad, bien hacia la libertad.

El segundo capítulo recoge solamente los apartados más significativos de la obra leviniana Umění překladu [El arte de la traducción], que hasta hoy ha gozado de cuatro ediciones en su lengua de origen (1963, 1983, 1998 y 2012). En 2011 se publicó la obra entera en inglés; la versión alemana salió a la luz al poco de su aparición en checo, o sea, en 1969; la rusa, en 1974 y la traducción al serbo-croata, en 1982. Una de las novedades de aquel entonces (1963) es el carácter comunicativo del proceso de la traducción: el texto original del autor es leído e interpretado, es decir descodificado por el traductor que lo reformula (recodifica) en su idioma, luego el texto traducido es leído y concretizado por el lector, lo que nos coloca ante una doble cadena de comunicación. Otro de los postulados de Levý que nos ofrece este capítulo son las tres fases de la labor del traductor, o sea, la comprensión, la interpretación y la reformulación del patrón o texto de partida, exigiendo esta última un elevado talento estilístico. Además, el autor reflexiona sobre las tendencias políticas y estéticas de una determinada época que influyen en la figura del traductor formando en él su "sujeto creador". Este capítulo incluye también otros conceptos como el de la traducción como género del arte, la doble norma de la traducción, la dualidad de la obra traducida, la relación ambigua con la literatura de partida, la tradición traductiva, la traducción "clásica" o las especificidades nacional e histórica, mencionando, por ejemplo, el trasvase de las variantes intralingüísticas. En cuanto a los procedimientos de trabajo del traductor, Levý distingue la traducción, la sustitución y la transcripción. Hablando de la poética de la traducción, el teórico checo distingue entre el estilo literario y traslativo, entre otros; establece una relación entre los términos conjunto y parte o idea y expresión. En las últimas páginas de este capítulo se estudia la traducción de los títulos.

En el tercer capítulo encontramos la versión abreviada del estudio Génesis de la obra literaria y su recepción, publicada póstumamente en la antología de textos levinianos Bude literární věda exaktní vědou? [¿Será la teoría literaria una ciencia exacta?] (1971) así como la síntesis de dos textos, en los cuales Levý analiza la génesis de la traducción como modelo de la creación de la obra literaria. Sus explicaciones parten del siguiente modelo: la traducción es un continuo proceso de decisiones que culmina en el trasvase, en el comunicar. Estas decisiones pueden ser, según afirma el autor, de índole "obligatoria" o "voluntaria" y "motivada" o "no motivada", sin embargo, siempre subordinadas a la norma estética que condiciona la elección del traductor.
El cuarto capítulo incluye un resumen del estudio Čapkovy překlady ve vývoji českého překladatelství a českého verše [Las traducciones de Karel Čapek en la evolución del arte de traducir y del verso checos] y subraya dos mensajes capitales para Levý: la influencia del trasvase en el desarrollo de la obra del traductor y la prioridad de la relación establecida entre el autor y el traductor.

El último capítulo que cierra el libro aporta el trabajo ¿Servirá la teoría de la traducción a los traductores? que se centra de nuevo en la figura del traductor y resume las principales líneas de pensamiento de Levý. El filólogo checo propone un análisis racional y objetivo basado en los principios procedentes de otras disciplinas, a saber la semántica, la psicolingüística, la antropología estructural entre otras, para investigar el proceso del trasvase.

Los traductores-editores, Králová y Cuenca, han facilitado al público hispanohablante el acceso a una de las reflexiones más inspiradoras en los estudios de la traducción y han contribuido así a la renovación metodológica del paisaje traductológico internacional.

Petra Vavroušová České vysoké učení technické/Universidad Politécnica Checa, Praga, República Checa

PÉlisse, Jérôme, Protais, Caroline, LARChet, Keltoume et Charrier, Emmanuel, dir. (2012): Des chiffres, des maux et des lettres: une sociologie de l'expertise judiciaire en économie, psychiatrie et traduction. Paris: Armand Colin, $274 \mathrm{p}$.

Cet ouvrage, paru dans la collection Recherches, décrit l'évolution du milieu des experts judiciaires en France à la suite de la réforme de 2004 visant leur reconnaissance, et s'intéresse plus précisément au cas des experts en économie, en psychiatrie et en traduction/interprétation. En effet, partant du constat que les experts sont omniprésents dans les médias, les auteurs se demandent d'une part si l'institution judiciaire recourt plus que jamais aux savoirs techniques et scientifiques, et d'autre part s'interrogent aussi sur la professionnalisation des experts. S'ils ne sont pas à même d'apporter un éclairage sur le premier point, leur analyse ${ }^{1} \mathrm{du}$ milieu des traducteurs/interprètes (TI) se révèle intéressante et d'actualité ${ }^{2}$.

Le rôle des TI: tout d'abord, les auteurs constatent que les TI sont différents des autres experts en ce qu'ils interviennent sur le plan procédural (la majeure partie du temps au pénal, viennent ensuite le civil et l'administratif) et qu'ils ne sont pas tant là pour donner leur opinion d'expert que pour faciliter la communication entre les différentes parties; ils se font la voix de l'autre, 